\title{
An alternative derivation of the minimal massive 3D gravity
}

\author{
Ahmet Baykal \\ Department of Physics, Faculty of Arts and Sciences, Niğde University, Bor Yolu, \\ 51240 Niğde, Turkey \\ E-mail: abaykal@nigde.edu.tr
}

\begin{abstract}
By using the algebra of exterior forms and the first order formalism with constraints, an alternative derivation of the field equations for the Minimal massive $3 \mathrm{D}$ gravity model is presented.

PACS numbers: $04.20 . \mathrm{Cv}, 04.60 . \mathrm{Kz}, 04.60 . \mathrm{Rt}$
\end{abstract}




\section{Introduction and geometrical preliminary}

The Minimal massive gravity (MMG) has recently been introduced in [1] as an alternative to the Topologically massive gravity (TMG) in three dimensions [2]. The field equations for MMG involve a particular quadratic-curvature terms obtained in a nontrivial way by extending the TMG Lagrangian. The MMG model has the same gravitational degree of freedom as the TMG has and the linearization of the metric field equations for MMG yield a single propagating massive spin-2 field. At the same time, the complicated issue of matter coupling to the MMG model, in the particular form of an ideal fluid, is studied in [3]. Compared to its cousin TMG, the new feature of the MMG model is the positivity of the central charge defined for the holographically dual conformal field theory on a three dimensional anti-de Sitter $\left(A d S_{3}\right)$ boundary. The hamiltonian analysis that follow from the MMG Lagrangian and the explicit computation of the dual conformal field theory charge are also provided along with the original derivation of the MMG equations [1]. The present brief report deals with an alternative derivation of the MMG field equations using a tensorial language.

The organization of the paper is as follows. After introducing the geometrical notation in the rest of the introductory section, the MMG field equations are derived in the following section. The derivation of the field equations given here provides further insight into the complicated system of field equations that follow from MMG Lagrangian. The paper ends with a short concluding section commenting on matter coupling to the MMG model. For convenience of the reader the basic geometrical definitions and quantities used below are summarized in the rest of the introductory section.

The geometrical notation for the exterior algebra required in the study of the MMG equations can be summarized as follows. The metric tensor has constant components relative to an orthonormal coframe, $g=\eta_{a b} e^{a} \otimes e^{b}$ with $\eta_{a b}=\operatorname{diag}(-++)$ and the Latin indices refer to an orthonormal coframe that can be expanded into coordinate coframe as $e^{a}=e_{\mu}^{a} d x^{\mu}$. The set of basis frame fields is $\left\{X_{a}\right\}$ and the abbreviation $i_{X_{a}} \equiv i_{a}$ is used for the contraction operator with respect to the basis frame field $X_{a}$.* denotes the Hodge dual operator acting on the basis forms, $* 1=e^{0} \wedge e^{1} \wedge e^{2}=\frac{1}{3 !} \epsilon_{a b c} e^{a b c}$ stands for the oriented volume element and $\epsilon_{a b c}$ is the permutation symbol in three dimensions. The abbreviations, for example, of the form $e^{a b} \equiv e^{a} \wedge e^{b}$ for the exterior products of basis 1-forms are used for the convenience of the notation. The first structure equation of Cartan reads

$$
\Theta^{a}=D(\omega) e^{a}=d e^{a}+\omega_{b}^{a} \wedge e^{b}
$$

The first Bianchi identity can be written in the form $D(\omega) \Theta^{a}=D^{2}(\omega) e^{a}=\Omega^{a}{ }_{b}(\omega) \wedge e^{b}$. $D(\omega)$ is the covariant exterior derivative operator for the connection 1-form $\omega^{a}{ }_{b}$, acting on tensor-valued forms, and a suitable definition and its relation to the covariant

derivative $\nabla_{a}$ can be found in [4]. The curvature 2-form $\Omega_{b}^{a}$ with $\Omega_{b}^{a}(\omega)=\frac{1}{2} R_{b c d}^{a}(\omega) e^{c d}$ satisfies the Cartan's second structure equation

$$
\Omega_{b}^{a}(\omega)=d \omega_{b}^{a}+\omega_{c}^{a} \wedge \omega_{b}^{c} .
$$


Ricci 1-form and the scalar curvature can be defined as the contractions $R^{a}=R_{b c}^{a c} e^{b} \equiv$ $R^{a}{ }_{b} e^{b}=i_{b} \Omega^{b a}$ and $R=i_{a} R^{a}$, respectively. It is convenient to define 3D Einstein 2-form in terms of curvature 2 -form as

$$
* G^{a}(\omega)=-\frac{1}{2} \Omega_{b c}(\omega) * e^{a b c}=-\frac{1}{2} \epsilon^{a b c} \Omega_{b c}(\omega)
$$

where $G^{a}(\omega)=R^{a}(\omega)-\frac{1}{2} R(\omega) e^{a}$ corresponding to the connection 1-form $\omega^{a}{ }_{b}$ in the same way as the Einstein 2-form defined in the Riemannian context. With a non-vanishing torsion, the first Bianchi identity takes the form $D(\omega) \Theta^{a}=\Omega_{{ }^{a}}{ }_{b}(\omega) \wedge e^{a}$ and consequently Einstein tensor is not be symmetrical in Riemann-Cartan geometry in general. The LeviCivita connection is denoted by $\Gamma_{b}^{a}$ relative to an orthonormal coframe. With vanishing nonmetricity 1-form, $Q_{a b}=-\frac{1}{2} D(\omega) \eta_{a b}=0$, the connection $\omega_{b}^{a}$ can be decomposed into the sum of a pseudo-Riemannian part and a contorsion part as

$$
\omega_{b}^{a}=\Gamma_{b}^{a}+K_{b}^{a}
$$

where the antisymmetric tensor-valued contorsion 1-forms $K_{a b}=-K_{b a}$ is related to the torsion 2-form by $\Theta^{a}=K_{b}^{a} \wedge e^{b}$. Consequently, the curvature 2-form $\Omega^{a}{ }_{b}(\omega)$ can be decomposed as

$$
\Omega^{a}{ }_{b}(\omega)=\Omega_{b}^{a}(\Gamma)+D(\Gamma) K_{b}^{a}+K_{c}^{a} \wedge K_{b}^{c} .
$$

The decomposition of the curvature 2-form given in Eq. (5) allows one to rewrite a given set of field equations in the Riemann-Cartan geometry context in terms of pseudoRiemannian quantities [5]. It is well-known that in 3D there is no Weyl 2-form and the curvature 2-form of a Riemann tensor can be expressed in terms of its contractions as

$$
\Omega^{a b}(\Gamma)=e^{a} \wedge L^{b}(\Gamma)-e^{b} \wedge L^{a}(\Gamma)
$$

where the Schouten 1-form $L_{a}=L_{a b} e^{b}$ can be defined in terms of Ricci 1-form and the scalar curvature as

$$
L^{a}=R^{a}-\frac{1}{4} R e^{a}
$$

( $\Gamma$-dependence is omitted from the equation for convenience). The Schouten 1-form can be used to derive the Cotton 2-form $C^{a}=\frac{1}{2} C^{a}{ }_{b c} e^{b c}$, explicitly one has $C^{a}=D(\Gamma) L^{a}(\Gamma)$.

The second Bianchi identity reads $D(\omega) \Omega^{a}{ }_{b}(\omega)=0$ and for the curvature 2form corresponding to the connection 1 -form $\Gamma_{b}^{a}$, the identity $D(\Gamma) \Omega_{b}^{a}(\Gamma)=0$ yields $e^{a} \wedge C^{b}=e^{b} \wedge C^{a}$ by using Eq. ([6]). The Cotton 2-form $C^{a}=\frac{1}{2} C^{a}{ }_{b c} e^{b c}$ can be related to the symmetric traceless Cotton tensor $C_{a b}=C_{b a}$ by the relation

$$
C_{a b}=i_{a} * C_{b} .
$$

By using the definition $C^{a}=D(\Gamma) L^{a}(\Gamma)$ and the relation $i_{a} D L_{b c}=\nabla_{a} L_{b c}$ in Eq. (8), one can obtain a more familiar expression for the Cotton tensor relative to an orthonormal coframe as

$$
C^{c d}=\epsilon^{a b c} \nabla_{a} L_{b}^{d}
$$

where $\nabla_{a}$ is covariant derivative corresponding to the Riemannian connection $\Gamma^{b}{ }_{c}$. 
The properties of the Cotton 2-form has been previously studied using the language of the tensor-valued exterior forms and for further properties of the Cotton tensor and Cotton 2-form, for TMG and the other related gravitational models, the reader is referred to [6].

\section{Minimal massive gravity Lagrangian and the field equations}

The field equations for the MMG model are derived from a Lagrangian 3-form which can be defined by a seemingly simple extension of the Lagrangian for TMG model with a cosmological constant. The MMG Lagrangian, that is introduced recently in [1], can explicitly be rewritten in terms of exterior forms as

$$
L_{M M G}=L_{T M G}+\frac{\alpha}{2} \lambda_{a} \wedge \lambda_{b} \wedge * e^{a b}
$$

where $\alpha$ is a coupling constant and the Lagrangian 3-form for the TMG Lagrangian [2] with a cosmological constant $\Lambda$ can be written in the form

$$
L_{T M G}=-\frac{\sigma}{2} \Omega_{a b} \wedge * e^{a b}+\frac{1}{4 \mu}\left(\omega^{a}{ }_{b} \wedge d \omega^{b}{ }_{a}+\frac{2}{3} \omega^{a}{ }_{b} \wedge \omega^{b}{ }_{c} \wedge \omega^{c}{ }_{a}\right)+\Lambda * 1+\lambda_{a} \wedge \Theta^{a} .
$$

In the TMG Lagrangian, the Einstein-Hilbert term is extended by the gravitational Chern-Simons term with constant $\mu$ and the auxiliary variable $\lambda^{a}=\lambda^{a}{ }_{b} e^{b}$ is a vectorvalued 1-form introduced to impose the vanishing torsion constraint for the TMG model. $\sigma$ is another constant. For the motivation for introducing the $\lambda^{2}$-coupling term in the MMG Lagrangian, the reader is referred to the original reference [1].

As it stands, the MMG Lagrangian depends on three gravitational variables,

$$
L_{M M G}=L_{M M G}\left[e^{a}, \omega_{b}^{a}, \lambda^{a}\right]
$$

and the field equations for the variables $\left\{e^{a}\right\},\left\{\omega_{b}^{a}\right\},\left\{\lambda^{a}\right\}$ can derived from a variational principle by using a first order formalism [6, 7]. In contrast to the original derivation using the dualized connection 1-form, the present derivation makes use of the usual connection 1-form $\omega^{a}{ }_{b}$ for the independent gravitational variable whose properties are defined in the preliminary section above.

One can show after some straightforward calculations in the exterior algebra that the total variational derivative of the Lagrangian (10) with respect to the independent variables is given by

$$
\begin{aligned}
\delta L_{M M G}= & \delta e_{a} \wedge\left(\sigma * G^{a}(\omega)+\Lambda * e^{a}+D(\omega) \lambda^{a}+\frac{\alpha}{2} \epsilon_{b c}^{a} \lambda^{b} \wedge \lambda^{c}\right) \\
& +\delta \omega_{a b} \wedge\left\{-\frac{\sigma}{2} D(\omega) * e^{a b}+\frac{1}{2 \mu} \Omega^{b a}(\omega)-\frac{1}{2}\left(e^{a} \wedge \lambda^{b}-e^{b} \wedge \lambda^{a}\right)\right\} \\
& +\delta \lambda_{a} \wedge\left(\Theta^{a}+\alpha \epsilon_{b c}^{a} e^{b} \wedge \lambda^{c}\right)
\end{aligned}
$$

up to a disregarded boundary term. Here $\delta$ denotes the variation of a quantity. For convenience, the technical details of the variational calculations leading to the important result (13) are given in the appendix. 
The terms on the right-hand side of the first line in (13), namely, the variational derivative with respect to the basis coframe 1-forms give the metric field equations for the MMG Lagrangian whereas the second the third lines yield the equations of motion for the gravitational variable $\omega_{a b}$ and the auxiliary variable $\lambda_{a}$ respectively.

It is convenient to start with the field equations for the auxiliary field 1-form $\lambda^{a}$. Instead of imposing vanishing torsion condition on the connection 1-forms $\omega_{a b}$, the equations $\delta L_{M M G} / \delta \lambda_{a} \equiv P^{a}=0$ yields a torsion in terms $\lambda^{a}$. Explicitly, by making use of the Cartan's first structure equations Eq. (1), the field equation

$$
\Theta^{a}+\alpha \epsilon_{b c}^{a} e^{b} \wedge \lambda^{c}=0
$$

can be rewritten in a more suggestive form as

$$
d e^{a}+\left(\omega_{b}^{a}-\alpha \epsilon_{b c}^{a} \lambda^{c}\right) \wedge e^{b}=0 .
$$

Consequently, the expression in the brackets in Eq. (15) satisfy the structure equations with vanishing torsion. Eq. (15) for the Lagrange multiplier 1-form help to decompose the connection 1-form $\omega_{b}^{a}$ into the Riemannian part denoted by $\Gamma_{b}^{a}$ defined by the second term in (15) and a contorsion part as

$$
\omega_{b}^{a}=\Gamma_{b}^{a}+\alpha \epsilon_{b c}^{a} \lambda^{c}
$$

and one can readily identify the contorsion 1 -form as $K_{b}^{a}=\alpha \epsilon_{b c}^{a} \lambda^{c}$. The decomposition of the connection 1-form (16) can be used to decompose the curvature 2-form as well. Using the identity given in Eq. (5), one finds

$$
\Omega^{a}{ }_{b}(\omega)=\Omega_{b}^{a}(\Gamma)+\alpha \epsilon_{b c}^{a} D(\Gamma) \lambda^{c}-\alpha^{2} \lambda^{a} \wedge \lambda_{b} .
$$

Likewise, the Einstein 2-forms also decompose as

$$
* G^{a}(\omega)=* G^{a}(\Gamma)-\alpha D(\Gamma) \lambda^{a}+\frac{\alpha^{2}}{2} \epsilon_{b c}^{a} \lambda^{b} \wedge \lambda^{c} .
$$

With the help of Eqs. (17) and (18), the connection equations can be expressed in terms of the Levi-Civita connection $\Gamma_{b}^{a}$. To this end, note first that by using the expression for the contorsion 1-form, one finds that the covariant exterior term can be written in terms of the auxiliary field 1-form as

$$
D(\omega) * e^{a b}=\epsilon_{c}^{a b} \Theta^{c}=\alpha\left(e^{a} \wedge \lambda^{b}-e^{b} \wedge \lambda^{a}\right) .
$$

Thus, by combining these results, the vacuum field equations for the connection 1-forms obtained by $\delta L_{M M G} / \delta \omega_{a b} \equiv S^{a b}=0$ can be rewritten in the form

$$
-\frac{1}{2 \mu} \Omega^{a b}(\Gamma)-\frac{\alpha}{2 \mu} \epsilon_{c}^{a b} D(\Gamma) \lambda^{c}+\frac{\alpha^{2}}{2 \mu} \lambda^{a} \wedge \lambda^{b}-\frac{1}{2}(1+\alpha \sigma)\left(e^{a} \wedge \lambda^{b}-e^{b} \wedge \lambda^{a}\right)=0(20)
$$

in terms of the Riemannian quantities.

In contrast to the TMG case with $\alpha=0$, in which $\lambda^{a}$ can uniquely be solved, the connection equations (20) are not a set of algebraic equations in the MMG case. To put it more precisely, owing to the presence of the term

$$
D(\Gamma) \lambda^{a}=d \lambda^{a}+\Gamma_{b}^{a} \wedge \lambda^{b}
$$


that contains the exterior derivatives of the auxiliary 1 -form field $\lambda^{a}$, the reduced equations for the connection 1 -forms yield a set of dynamic equations for the auxiliary field $\lambda^{a}$. In the TMG case $(\alpha=0)$ it is easy to find that the algebraic equation (20) for $\lambda^{a}$ has a unique solution

$$
\lambda^{a}=-\frac{1}{\mu} L^{a}(\Gamma)
$$

with the help of the curvature identity (6) $)$. On the other hand, for $\alpha \neq 0$, i.e., for the MMG case, it is difficult to find the general solution for $\lambda^{a}$ in a closed form using the reduced connection equations given in (20). Consequently, it is not possible to eliminate the auxiliary vector-valued 1-form $\lambda^{a}$ from the metric equations obtained by the coframe variations, $\delta L_{M M G} / \delta e^{a} \equiv * E^{a}=0$, in favor of the remaining gravitational variables. Thus, without a closed expression for the Lagrange multiplier 1-form $\lambda^{a}$ in terms of other fields, it is not possible to reduce the MMG Lagrangian (10) to a form $L_{M M G-\text { red. }}\left[\Gamma_{b}^{a}, e^{a}\right]$ by a back-substitution.

With the benefit of the hindsight and the fact that for $\alpha=0$ the solution is of the form (22), one can try a simple "ansatz" proportional to the Schouten 1-form. However, assuming a non-vanishing cosmological constant, a suitable ansatz with two adjustable constant $p$ and $q$, is of a slightly more general form:

$$
\lambda^{a} \equiv p L^{a}(\Gamma)+q e^{a} .
$$

By plugging the ansatz (23) into Eq. (20), by making use of the identity (6), the definition of Cotton 2-form, $D(\Gamma) L^{a}(\Gamma)=C^{a}$, and the torsion-free condition, $D(\Gamma) e^{a}=0$ for $\Gamma$, one finds

$$
-\frac{1}{2} A \Omega^{a b}(\Gamma)+\frac{1}{2} B \epsilon_{c}^{a b} C^{c}+\frac{1}{2} E e^{a} \wedge e^{b}+\frac{1}{2} F L^{a}(\Gamma) \wedge L^{b}(\Gamma)=0
$$

with the constants $A, B, E, F$ that can be expressed in terms of the constants $p, q$ and the constants in the MMG Lagrangian as

$$
\begin{array}{lll}
A=\frac{1}{\mu}\left(\alpha^{2} p q-1\right)-p(1+\alpha \sigma), & B=-\frac{\alpha}{\mu}, \\
E=\frac{\alpha^{2} q^{2}}{\mu}-2 q(1+\alpha \sigma), & F=\frac{p^{2} \alpha^{2}}{\mu} .
\end{array}
$$

By multiplying Eq. (24) with a permutation symbol and taking the definition of the Einstein 2-form (3) into account, Eq. (24) can finally be brought to the form, similar to the equation for basis coframe 1-forms, as

$$
A * G^{a}+B C^{a}+E * e^{a}+\frac{1}{2} F \epsilon_{b c}^{a} L^{b} \wedge L^{c}=0
$$

with the obvious $\Gamma$-dependence of the curvature terms omitted. It is possible to show that Eq. (27) is equivalent to the expression for the MMG field equations given in the original form relative to a coordinate basis [1]. In particular, the quadratic-curvature terms can be expressed with a suitable vector-valued 2-form defined by

$$
J^{a} \equiv \frac{1}{2} \epsilon_{b c}^{a} L^{b} \wedge L^{c}
$$


in the notation used above. The original definition of the quadratic-curvature term corresponds to the coordinate components of the expression $J^{a b} \equiv i^{a} * J^{b}$. The first three terms in (27) are the terms appearing in the TMG field equation (with cosmological constant) with shifted constants. The last term in (27) is involves the quadraticcurvature terms, which is a consequence of the MMG field equations and as well as the ansatz (23). As it has been noted in the original construction [1], note that the trace of the quadratic-curvature term yields the quadratic-curvature part of the New massive gravity Lagrangian [8] up to a constant multiple. By a straightforward calculation, one can show explicitly that

$$
e^{a} \wedge J_{a}=\frac{1}{2} L_{a} \wedge L_{b} \wedge * e^{a b}=-\frac{1}{4}\left(R^{a} \wedge * R_{a}-\frac{3}{8} R^{2} * 1\right)
$$

where the curvature components in the expression correspond to the curvature of a Levi-Civita connection $\Gamma^{a}{ }_{b}$.

Finally, the significance of the ansatz (23) becomes more pronounced after one rewrites the coframe (equivalently, the metric) equations

$$
\sigma * G^{a}(\omega)+\Lambda * e^{a}+D(\omega) \lambda^{a}+\frac{\alpha}{2} \epsilon_{b c}^{a} \lambda^{b} \wedge \lambda^{c}=0
$$

in terms of the Riemannian quantities as well. By using the decomposition formulas (17) and (18), one finds that

$$
\sigma * G^{a}(\Gamma)+(1-\alpha \sigma) D(\Gamma) \lambda^{a}+\Lambda * e^{a}+\frac{1}{2} \alpha(3+\alpha \sigma) \epsilon_{b c}^{a} \lambda^{b} \wedge \lambda^{c}=0 .
$$

Now, assuming that the coefficients the terms in Eq. (31) do not vanish identically, one can now plug in the ansatz into Eq. (31). Explicitly, by plugging the ansatz (23) into the coframe equations, and subsequently using the curvature identity (6) and the definition of the quadratic-curvature term $J^{a}$ given in Eq. (28), one eventually finds that Eq. (31) can be rewritten in the form

$$
\begin{aligned}
{[\sigma-p q \alpha(3+\alpha \sigma)] * G^{a}+p(1-\alpha \sigma) C^{a}+[\Lambda+} & \left.q^{2} \alpha(3+\alpha \sigma)\right] * e^{a} \\
& +p^{2} \alpha(3+\alpha \sigma) J^{a}=0
\end{aligned}
$$

with the $\Gamma$-dependence of the curvature terms omitted.

By comparison, one can see that Eq. (32) has the same form as that of Eq. (27) up to the constants multiplying each term. The ansatz in Eq. (23) is unique in the sense that it renders the coframe and the connection equations identical up to a duality provided that the constants in the Eqs. (27) and (32) are identified accordingly.

Explicitly, in the present notation and relative to an orthonormal coframe, MMG equations (using the constants with which they are defined originally) for the coframe and the connection 1-forms take the form

$$
\sigma * G^{a}+\Lambda_{0} * e^{a}+\frac{1}{\mu} C^{a}+\frac{\gamma}{\mu^{2}} J^{a}=0
$$

that is an equation for 2-forms in a form in line with, for example, the form of the TMG field equations given in [6] and [9].

Finally, the following remarks are in order regarding the derivation of the MMG field equations. 
(i) It is worth emphasizing that, without the assumption of the ansatz (23) for $\lambda^{a}$, the field equations for the independent coframe and the connection 1-forms can be expressed in terms of pseudo-Riemannian quantities with the help of the constraint (14). The connection equations (20) can be regarded as general set of dynamic equations for the auxiliary vector-valued 1-form field $\lambda^{a}$ and for the particular ansatz (23), the MMG field equations simplify considerably. To put it in mathematical terms, evaluated using the ansatz (23) in terms of a Levi-Civita connection, the coframe equations $\delta L_{M M G} / \delta e_{a}=* E^{a}$ can be made equal to the dual of the connection equations $\delta L_{M M G} / \delta \omega_{a b}=S^{a b}$ as

$$
\left.\frac{1}{2} \epsilon_{b c}^{a} S^{b c}\right|_{\text {ansatz }}=\left.* E^{a}\right|_{\text {ansatz }}
$$

by adjusting the parameters of the MMG model and the ansatz.

(ii) Even though the MMG Lagrangian (10) contains a constraint term imposing the vanishing torsion condition on the independent connection 1-form, it leads to a Riemann-Cartan type geometry [5] and the MMG model is unique in the sense that, written in terms of Riemannian quantities, the equations for the connection and coframe 1-forms can be identified by a particular choice of the auxiliary field variable. This insight for the field equations of the MMG model seems to be new. In $D>3$ dimensions, the construction will not work for the reason that the derivation makes the essential use of the curvature identity (6) peculiar to three dimensions.

(iii) In contrast to the original derivation of the MMG field equations that makes use of the dualized connection and the curvature forms, the relation given in Eq. (2.11) in [1, namely, $e \cdot h=e^{a} h^{b} \eta_{a b}=0$ is nowhere assumed to hold in the derivation of the field equations obtained from Eq. (13), but it is a simplifying property encoded in the "ansatz" given in Eq. (23). As it is emphasized above, a general closed expression for $\lambda^{a}$ in terms of the other gravitational variables is not available, and hence it is not possible to eliminate the auxiliary variable $\lambda^{a}$ from the metric equations or to reexpress the MMG Lagrangian 3-form in terms of the Riemannian quantities.

(iv) Throughout the above derivation, the tensorial manipulations are preformed relative to an orthonormal coframe yielding the field equations relative to a orthonormal basis in a unified manner.

\section{Concluding comments}

With regard to the matter coupling of the MMG, it may be more convenient to consider the MMG field equations in terms of the variables $e^{a}, \omega^{a}{ }_{b}, \lambda^{a}$ before casting the field equations in a form expressed in terms of the pseudo-Riemannian quantities.

It is possible to show that, in the first order formalism with independent connection and coframe forms, the diffeomorphism invariance of a gravitational model leads to the more general differential identity [10], involving the covariant exterior derivative 
of the coframe equations. Explicitly, for a general Lagrangian of the form $L=$ $L\left[e^{a}, \omega_{b}^{a}, \Theta^{a}, \Omega^{a}{ }_{b}\right]$ the invariance under a diffeomorphism $\phi$ can be expressed as

$$
\left(\phi^{*} L\right)\left[e^{a}, \omega_{b}^{a}, \Theta^{a}, \Omega_{b}^{a}\right]=L\left[\phi^{*} e^{a}, \phi^{*} \omega_{b}^{a}, \phi^{*} \Theta^{a}, \phi^{*} \Omega^{a}{ }_{b}\right]
$$

where $\phi^{*}$ stands for induced pullback map that $\phi$ generates on the tensor-valued $p$ forms. In particular, if one assumes that $\phi$ is generated by a vector field $Z$ depending on a parameter, say $t$, then taking the derivative of (35) with respect to the parameter $t$, and using the definition of the Lie derivative $\mathcal{L}_{Z}$, one readily finds

$$
\mathcal{L}_{Z} L=\left(\mathcal{L}_{Z} e^{a}\right) \wedge * E^{a}+\left(\mathcal{L}_{Z} \omega^{a}{ }_{b}\right) \wedge S^{a b}
$$

where an exact 3-form on the right-hand side is discarded. Furthermore, by using the Cartan's formula, $\mathcal{L}_{Z}=d i_{Z}+i_{Z} d$, for Lie derivative with respect to the vector field $Z=Z^{a} X_{a}$, and the Cartan's structure equations, one can simplify the expression in Eq. (36) to the form

$$
\begin{aligned}
\left(\mathcal{L}_{Z} e^{a}\right) \wedge * E^{a}+\left(\mathcal{L}_{Z} \omega^{a}{ }_{b}\right) \wedge S^{a b}= & -\left(i_{Z} e^{a}\right) D * E_{a}+i_{Z} \Theta_{a} \wedge * E^{a}+\left(i_{Z} \Omega_{a b}\right) \wedge S^{a b} \\
& +\left(i_{Z} \omega_{a b}\right)\left\{\frac{1}{2}\left(e^{a} \wedge * E^{b}-e^{b} \wedge * E^{a}\right)-D S^{a b}\right\} \\
& +d\left\{\left(i_{Z} e^{a}\right) * E_{a}+\left(i_{Z} \omega_{a b}\right) S^{a b}\right\} .
\end{aligned}
$$

Finally, assuming that the boundary term vanishes, and setting the coefficients of $Z^{a}$ and $i_{Z} \omega_{a b}$ separately in (37), one ends up with the identities

$$
\begin{aligned}
& D * E_{a}=\left(i_{a} \Theta_{b}\right) \wedge * E^{b}+\left(i_{a} \Omega_{b c}\right) \wedge S^{b c}, \\
& D S^{a b}=\frac{1}{2}\left(e^{a} \wedge * E^{b}-e^{b} \wedge * E^{a}\right),
\end{aligned}
$$

respectively.

In the above framework, the diffeomorphism invariance of a general Lagrangian generates the generalized differential identity given in Eq. (38) that reduces $D * E^{a}=0$ for the pseudo-Riemannian case whereas the identity in Eq. (39) resulting from the invariance under coframe rotations (local Lorentz invariance) reduces to the symmetry property $E_{a b}=E_{b a}$ expressed in the form $e^{a} \wedge * E^{b}-e^{b} \wedge * E^{a}=0$.

For the particular case of the MMG Lagrangian, the implementation of the auxiliary field $\lambda^{a}$ and the ansatz (23) into the generalized differential Bianchi identity (38) obtained by the Noether procedure requires further scrutiny. In this regard, the first order formalism and Riemann-Cartan geometry may provide an alternative mathematical framework in the construction of consistent minimal matter coupling to the MMG model as well.

\section{Acknowledgments}

The author would like to thank O. Teoman Turgut and Özgür Delice for instructive correspondences and help. 


\section{Appendix}

In the first order formalism, the independent gravitational variables $\left\{e^{a}\right\},\left\{\omega^{a}{ }_{b}\right\}$ and their first order derivatives $\left\{d e^{a}\right\},\left\{d \omega_{b}^{a}\right\}$ are allowed in the Lagrangian 3-form. The derivatives of the variables appear only through tensorial quantities $\Theta^{a}$ and $\Omega^{a}{ }_{b}$, respectively. Hence, it is convenient to assume that $L_{M M G}=L_{M M G}\left[e^{a}, \omega^{a}{ }_{b}, \Theta^{a}, \Omega^{a}{ }_{b}, \lambda^{a}\right]$ where the Lagrange multiplier vector-valued 1 -form $\lambda^{a}$ is an auxiliary variable and impose vanishing torsion constraint for the connection in the $L_{T M G}$ Lagrangian.

The field equations can be obtained by the principle of stationary action, embodied in the variational equation $\delta I_{M M G}=0$. For the MMG model, the action functional is given by the integral of the Lagrangian 3 -form as

$$
I_{M M G}=\int_{U} L_{M M G}=\int_{U} L_{T M G}+\frac{\alpha}{2} \int_{U} \lambda_{a} \wedge \lambda_{b} \wedge * e^{a b}
$$

where $U \subset M$ is an open subset on some chart defined on a $(2+1)$-dimensional RiemannCartan manifold $M$.

For convenience of the notation, the integral sign will be omitted and the variational derivative of the Lagrangian 3-form will be considered. By using the product rule for the variational derivative, $\delta L_{T M G}$ can explicitly be written as

$$
\begin{aligned}
\delta L_{T M G}= & -\frac{\sigma}{2} \delta \Omega_{a b} \wedge * e^{a b}-\frac{\sigma}{2} \Omega_{a b} \wedge \delta * e^{a b}+\delta \lambda_{a} \wedge \Theta^{a}+\lambda_{a} \wedge \delta \Theta^{a}+\Lambda \delta * 1 \\
& +\frac{1}{4 \mu}\left(\delta \omega_{a b} \wedge d \omega^{b a}+\omega_{a b} \wedge d \delta \omega^{b a}+2 \delta \omega^{a}{ }_{b} \wedge \omega_{c}^{b} \wedge \omega_{a}^{c}{ }_{a}\right)
\end{aligned}
$$

where the cyclic property $\delta \omega^{a}{ }_{b} \wedge \omega^{b}{ }_{c} \wedge \omega^{c}{ }_{a}=\omega^{c}{ }_{a} \wedge \delta \omega^{a}{ }_{b} \wedge \omega^{b}{ }_{c}=\omega^{b}{ }_{c} \wedge \omega^{c}{ }_{a} \wedge \delta \omega^{a}{ }_{b}$ is used to derive the expression in the second line. To evaluate the variational derivative in Eq. (41) further, it is convenient to recall the following variational derivatives for various tensor-valued forms:

$$
\begin{aligned}
& \delta * e^{a b}=\epsilon^{a b}{ }_{c} \delta e^{c}=\delta e^{c} * e^{a b}{ }_{c} \\
& \delta * 1=\frac{1}{6} \epsilon_{a b c}\left(\delta e^{a} \wedge e^{b} \wedge e^{c}+e^{a} \wedge \delta e^{b} \wedge e^{c}+e^{a} \wedge e^{b} \wedge \delta e^{c}\right)=\delta e^{a} \wedge * e_{a}( \\
& \delta \Theta^{a}=\delta d e^{a}+\delta \omega^{a}{ }_{b} \wedge e^{b}+\omega^{a}{ }_{b} \wedge \delta e^{b}=D \delta e^{a}-\delta e^{b} \wedge \omega^{a}{ }_{b} \\
& \delta \Omega^{a}{ }_{b}=\delta d \omega^{a}{ }_{b}+\delta \omega^{a}{ }_{c} \wedge \omega^{c}{ }_{b}+\omega^{a}{ }_{c} \wedge \delta \omega^{c}{ }_{b}=D \delta \omega^{a}{ }_{b} .
\end{aligned}
$$

Note that the variational derivative $\delta$ commutes with the exterior derivative $d$, however, it does not commute with the covariant exterior derivative $D$ and the Hodge dual. $\delta \omega^{a b}$ is a tensor and thus the covariant exterior derivative $D \delta \omega^{a b}$ can be defined as in the expression on the right-hand side of Eq. (45).

By using the variational formulas given in Eqs. (42)-(45) and the Cartan's structure equation (2), the total variational derivative $\delta L_{T M G}$ with respect to the independent connection, coframe and auxiliary 1-forms can explicitly be expressed as

$$
\begin{aligned}
\delta L_{T M G}= & -\frac{\sigma}{2} \delta \omega_{a b} \wedge D * e^{a b}-\delta e^{c} \wedge \frac{\sigma}{2} \Omega_{a b} \wedge * e^{a b}{ }_{c}+\delta e^{a} \wedge \Lambda * e_{a} \\
& +\delta e_{a} \wedge D \lambda^{a}-\delta \omega_{a b} \wedge \frac{1}{2}\left(e^{a} \wedge \lambda^{b}-e^{b} \wedge \lambda^{a}\right)+\frac{1}{2 \mu} \delta \omega_{a b} \wedge \Omega^{b a}
\end{aligned}
$$




$$
+\delta \lambda_{a} \wedge \Theta^{a}+d\left(-\frac{\sigma}{2} \delta \omega_{a b} \wedge * e^{a b}+\delta e_{a} \wedge \lambda^{a}+\frac{1}{2 \mu} \delta \omega_{a b} \wedge \omega^{b a}\right)
$$

where $D$ in the variational expression denotes the covariant exterior derivative with respect to the independent connection 1-form, denoted by $D(\omega)$ in the main text. The boundary term can be made to vanish by making use of the Stokes' theorem and assuming that $\left.\delta e^{a}\right|_{\partial U}=0$ and $\left.\delta \omega_{a b}\right|_{\partial U}=0$. Since $\omega_{a b}$ is assumed to be metriccompatible, $-D \eta_{a b}=\omega_{a b}+\omega_{b a}=0$ and likewise the variations satisfy $\delta \omega_{a b}+\delta \omega_{b a}=0$. Consequently the coefficient of $\delta \omega_{a b}$ is to be antisymmetrized with respect to the indices $a$ and $b$.

The variational derivative of the $\lambda^{2}$-term contributes to the field equations for both the coframe and the auxiliary field $\lambda^{a}$. Explicitly, the total variational derivative of the $\lambda^{2}$-term reads

$$
\begin{aligned}
\delta\left(\lambda_{a} \wedge \lambda_{b} \wedge * e^{a b}\right) & =\delta \lambda_{a} \wedge \lambda_{b} \wedge * e^{a b}+\lambda_{a} \wedge \delta \lambda_{b} \wedge * e^{a b}+\lambda_{a} \wedge \lambda_{b} \wedge \delta * e^{a b} \\
& =2 \delta \lambda_{a} \wedge \lambda_{b} \wedge * e^{a b}+\delta e^{c} \wedge \epsilon_{a b c} \lambda^{a} \wedge \lambda^{b}
\end{aligned}
$$

By combining the above expression for the variational derivatives, one eventually ends up with an expression of the form

$$
\delta L_{M M G}=\delta e_{a} \wedge * E^{a}+\delta \omega_{a b} \wedge S^{a b}+\delta \lambda_{a} \wedge P^{a}
$$

up to a discarded exact 3-form. The explicit expressions for the tensor-valued forms on the right-hand side read

$$
\begin{aligned}
& \frac{\delta L_{M M G}}{\delta e_{a}} \equiv * E^{a}=\sigma * G^{a}+\Lambda * e^{a}+D \lambda^{a}+\frac{\alpha}{2} \epsilon_{b c}^{a} \lambda^{b} \wedge \lambda^{c}, \\
& \frac{\delta L_{M M G}}{\delta \omega_{a b}} \equiv S^{a b}=-\frac{\sigma}{2} D * e^{a b}+\frac{1}{2 \mu} \Omega^{b a}-\frac{1}{2}\left(e^{a} \wedge \lambda^{b}-e^{b} \wedge \lambda^{a}\right), \\
& \frac{\delta L_{M M G}}{\delta \lambda_{a}} \equiv P^{a}=\Theta^{a}+\alpha \epsilon_{b c}^{a} e^{b} \wedge \lambda^{c},
\end{aligned}
$$

where $E_{a}=E_{a b} e^{b}$ is a vector-valued 1-form, $S^{a b}=-S^{b a}$ is a antisymmetric tensorvalued 2 -form, whereas $P^{a}$ is a vector-valued 2 -form. The constraint equation $P^{a}=0$ leads to a non-vanishing torsion expressed in terms of $\lambda_{a}$ and it is difficult to eliminate $\lambda_{a}$ from the coframe equations $* E^{a}=0$ by first solving the connection equations $S^{a b}=0$ for $\lambda_{a}$ and taking the constraint into the account.

\section{References}

[1] Bergshoeff E, Hohm O, Merbis W, Routh A J, Townsend P K 2014 Class. Quantum Grav. 31 145008 (arXiv:1404.2867 [hep-th]).

[2] Deser S, Jackiw R, Templeton S 1982 Ann. Phys. (NY) 140372.

[3] Arvanitakis A S, Routh A J, Townsend P K 2014 Preprint, arXiv:1407.1264 [hep-th].

[4] Benn I M, Tucker R W 1987 An introduction to spinors and geometry with applications in physics, (IOP Publishing Ltd., Bristol) p. 199.

[5] Hehl F W, von der Heyde P, Kerlick G D and Nester J M 1976 Rev. Mod. Phys. 48393.

[6] García A A, Hehl F W, Heinicke C, and Macías A 2004 Class. Quantum Grav. 211099.

[7] Baykal A, Delice Ö 2011 Class. Quantum Grav. 28015014 (arXiv:1012.4246 [gr-qc]) 
[8] Bergshoeff E A, Hohm O and Townsend P K 2009 Phys. Rev. Lett. 102 201301; Bergshoeff E A, Hohm O and Townsend P K 2009 Phys. Rev. D 79124042.

[9] Dereli T, Tucker R W 1998 Class. Quantum Grav. 5951.

[10] Kopczyński W 1990 Ann. Phys. 203308. 